\title{
Moving towards a molecular understanding of pain management
}

\section{The future role of molecular medicine in pharmacologic treatment of patient pain}

Logan Van Nynatten, Shafaz Veettil

\section{ABSTRACT}

The management of chronic pain remains a challenging area in the practise of medicine. As our population ages, the incidence and prevalence of those living with chronic pain continues to increase. Hence, there is need for methods that promote optimal pain management. One promising avenue is that of "personalized and molecular pain management". Indeed, a variety of genetic and molecular factors have been shown to impact metabolism of narcotics, limiting drug effectiveness. Furthermore, the prominence of polypharmacy can complicate the action of pain medications. Additional laboratory and diagnostic tests may be of benefit for risk stratifying patients at high risk of abusing pain medications from those at lower risk. Combining this with physician worry of worsening the opioid addiction crisis in North America via prescribing narcotics, there remains great pressure on physicians to limit their use of narcotics. Unfortunately, this may result in patients who are suitable candidates for prescription opioids receiving inadequate pharmaceutical treatment to complement non-pharmacological interventions. Moving forward, the implementation of molecular medicine approaches to pain management may provide unique solutions to these challenges.

\section{INTRODUCTION}

The diagnosis and treatment plans for chronic pain - often defined as pain lasting greater than 3 months - remains a challenging topic within medicine. Although we have the means and knowledge to treat pain, many Canadians suffer from inadequate pain treatment. A Canadian survey in 2012 found $29 \%$ of adults reported continuous or intermittent pain lasting six months or longer. ${ }^{1}$ Of these individuals, $88 \%$ rated their pain as a 4 or higher on a ten-point scale. ${ }^{1}$ The prevalence of chronic pain was also shown to increase with age as $22 \%$ of those aged $18-34$ and $39 \%$ of those aged 55 years or older reported chronic pain. ${ }^{2}$ Females also had a $4 \%$ higher prevalence than males. ${ }^{2}$ Other Canadian studies have found similar results. ${ }^{2,3}$ Indeed, pain is the most common reason patients seek healthcare, and accounts for approximately $78 \%$ of emergency department visits. ${ }^{4}$ However, large multicentre studies continue to demonstrate high pain intensity, and suboptimal pain management experienced by patients in Canada. ${ }^{4}$ Hence, the question becomes how do we adequately manage chronic pain in a responsible way as to prevent abuse of prescription narcotics? One promising avenue is that of "personalized and molecular pain management" to guide pharmacologic treatment within molecular medicine.

\section{PERSONALIZED MEDICINE AND PAIN MANAGEMENT}

Personalized medicine pain management refers to the concept of optimizing medication types and dosage based on individual patient genetic and molecular variation. ${ }^{5}$ The use of personalized medicine is already being implemented in a variety of other medical fields, such as oncology. It is possible to risk stratify tumors based on the pattern of protein expression and treat accordingly. For example, breast cancers expressing the HER2 ligand can be treated with trastuzumab, gastrointestinal tumors overexpressing CD117 are responsive to tyrosine kinase inhibitors, and non-small lung cancer expressing PD-L1 suppressing the anti-cancer immune response can be blocked with pembrolizumab. ${ }^{6-8}$ These are agents that work to specifically limit the molecular pathways conferring growth advantages to specific tumors in specific patients. Similarly, we should be able to apply molecular approaches to optimize treatment of acute and chronic pain. Ideally, we could identify pathways or protein polymorphisms that either enhance or limit the effectiveness of pain medications for individual patients. Although the implementation of using patient genetic polymorphisms and gene copy number of drug metabolizing enzymes is still a few years away in premise, the rapid expansion of genome wide association studies and molecular medicine puts personalized pain management well within reach.

\section{METABOLIC FACTORS IMPACTING PAIN MANAGEMENT}

Cytochrome P450 (CYP) enzymes provide over $80 \%$ of phase I drug metabolism. As one can imagine, gene polymorphisms, deletions, or duplications can result in significant qualitative and quantitative variation in CYP enzyme mRNA and protein expression impacting enzymatic function. In particular, CYP2D6 - of which there are greater than 50 allelic variants reported metabolizes a variety of frequently used drugs including betablockers, antiarrhythmics, anti-depressants, neuroleptics, and critically, analgesics such as codeine, dihydrocodeine, hydrocodone, oxycodone and tramadol. ${ }^{9}$ As such, variation just in this enzyme alone can produce patients who are poor, intermediate, extensive and ultrarapid metabolizers of narcotics - something which is rarely considered in the office of a general medical practitioner. Furthermore, fluctuations in drug metabolism and the prevalence of such polymorphisms vary between ethnicities, with $3-5 \%$ of the European, 10-12\% of Mediterranean, 21\% of Saudi Arabian, and 29\% 
of the Ethiopian population being ultrarapid metabolizers of such narcaotics. ${ }^{9}$ Such differences highlight how the impact of genetic variation on drug metabolism may be worthwhile considering in clinical practise.

There are multiple examples of other genetic variations potentially impacting drug metabolism and hence the patient's experience living with chronic pain. NSAIDs, or non-steroidal anti-inflammatory drugs, are a common class of drugs used in the multimodal approach to pain management which are largely metabolized by CYP2C9 and CYP2C8 enzymes. ${ }^{9}$ At least 33 genetic variants have been reported for CYP2C9, again varying amongst ethnicities. ${ }^{9}$ Specifically, the two missense mutations more common in the Caucasian population, which include switching $\operatorname{Arg}_{144}$ to a cysteine and Ile 359 to a leucine result in decreased enzymatic activity. ${ }^{10}$ Such polymorphisms could affect the metabolism of widely used drugs such as ibuprofen, naproxen, and celecoxib. ${ }^{9}$ The presence of the $\mathrm{G}_{472} \mathrm{~A}$ polymorphism in Catechol$\mathrm{O}$-Methyltransferase impacts dopaminergic and adrenergic neurotransmission through a 3-4 fold decrease in the metabolism of catecholamine, having important consequences for drugs commonly prescribed for chronic cancer pain. ${ }^{11}$ The metabolism of many drugs is impacted by several CYPs, such as tricyclic antidepressants (TCAs) often used in treating neuropathic pain which are metabolized by both CYP2D6 and CYP2C19. ${ }^{12}$ Hence the serum plasma levels of TCAs can be impacted by polymorphisms in both enzymes. ${ }^{11}$ Cumulatively, these findings highlight how such subtle molecular variations can potentially impact the effectiveness of pharmacologic therapy, thereby impacting the degree to which pain relief may be experienced by patients. Incorporating such knowledge of genetic variations when developing treatment plans for patients may represent a great opportunity for optimizing treatment on an individual patient-to-patient basis.

\section{POLYPHARMACY AND CHRONIC PAIN MANAGEMENT}

Even taking into consideration the genetic factors impacting pain medication efficacy, metabolism, and duration of action, it is equally important to consider the other medications that the patient has been prescribed that could impact metabolism of narcotics, and hence the patient's pain experience. Polypharmacy - the use of multiple drugs in the treatment of medical conditions - is quite prevalent in patient care today. It is not uncommon to see upwards of a dozen medications being taken by an individual chronically ill patient every day. ${ }^{13,14}$ The molecular targets of such drugs can alter cellular metabolism to both increase or decrease the efficacy of drugs prescribed for pain management. As an example, the $\mathrm{ABCB} 1 / \mathrm{MDR} 1$ gene encodes an efflux transporter to remove certain substances from cells, and affects bioavailability of fentanyl, morphine, and related pain medication derivatives. ${ }^{15}$ There are common drugs that both induce (ex atorvastatin, cyclosporine, erythromycin, itraconazole, methadone, verapamil, tamoxifen) and inhibit (ex dexamethasone, morphine, retinoic acid) MDR1 activity. ${ }^{16}$ Prescription of drugs that have competing and synergistic effects on the bioavailability of drugs such as morphine and fentanyl are rarely considered and are likely a component as to why individuals may experience inadequate pain relief at doses physicians feel should be more than adequate to relieve pain. Combining this with the natural polymorphisms observed in MDR1, one can imagine how a high-throughput screening of drug interactions and patient genetic variation could be a useful tool to help specifically manage patients with chronic pain that are responding to treatment poorly.

Indeed, there is much work being done to try and develop such a high-throughput screening system, however it is not without its challenges. Most of these methods are based on either in vitro chemical reactions or in silico simulations of drug interactions. ${ }^{17}$ Of course, this does not fully account for the dynamic molecular interactions which occur in vivo impacting drug-drug interactions. Regardless, one main assay used to investigate drugdrug interactions in pre-clinical drug discovery, are in vitro high throughput fluorometic screening methods. Largely this works via multiwell plates containing CYP enzymes which metabolize a substrate into a fluorescent product, which can be detected. ${ }^{17}$ The addition of single or multiple drugs can be added to these wells, and their isolated or combined impact on CYP enzyme metabolism can be recorded based on fluorescence variation. ${ }^{17}$ At the in silico level, computer algorithms are in development that strive to predict drugdrug interactions based on molecular paramaters. ${ }^{17}$ We propose that as these assays become more robust, the data collected from such high throughput analysis could be consolidated to generate a reference database where clinicians could refer to and make note of combinations of drugs that either maximise or limit the effect they are trying to achieve, for those patients who appear to show a lack of response to treatment.

\section{MOVING TOWARDS A MOLECULAR UNDERSTANDING OF NARCOTIC ABUSE}

Undeniably, there is an opioid abuse and addiction epidemic in London, Ontario, as well as across Canada. ${ }^{24}$ The Public Health Agency of Canada reported that at least 2458 Canadians died from opioid related addiction overdoses in 2016. ${ }^{24}$ Although opioid addiction is impacted by psychosocial and environmental factors, there may also be some additional genetic factors placing one at higher risk for addiction. It has been reported that polymorphisms of the mu-opioid receptor (A118G) and of the catechol-omethyltransferase gene (V158M) may alter analgesic responsiveness and opioid abuse risk. ${ }^{18,19}$ Additionally, although the mu-opioid receptor (MOR) mediates most of the analgesic actions of opioids, the kappa-opioid receptor (KOR) has been suggested to be involved in limiting MOR analgesic tolerance, dependence, and withdrawal symptoms. Certain polymorphisms of the KOR gene (OPRK1) have demonstrated a significant association with populations of heroin addicts, and sequence variations in the MOR gene (OPRM1) have been found to be associated with heroin addiction in humans. ${ }^{18,19}$ Similar associations have been found with polymorphisms in the PYDN and ABCB1 gene families. ${ }^{20}$ It remains unclear as to whether such polymorphisms are predisposing factors for addiction or are the consequence of the development of drug resistance. As 
these and future associations become more clearly elucidated and understood, they may hold tremendous promise for the field of pain management. Indeed, the vast reason opioids are under-prescribed for chronic pain management in certain populations is physicians' fear they will be stimulating opioid abuse and addiction. ${ }^{21}$ This is reasonable considering the majority of those who abuse opioids have their first exposure to the drugs from physicians. Currently, there is no effective laboratory test to risk stratify those patients who are at higher risk for addiction, abuse and opioid dependence from those who are not. Such a test would be useful to complement the widely utilized Opioid Risk Tool that currently exists as a survey screening method for determining risk of opioid abuse based on various patient psychosocial risk factors. ${ }^{22}$ Although addiction is in no way solely a genetic problem, using such personalized medicine approaches may help contribute to the generation of more effective criteria in prescription and dosing of narcotics.

\section{CONCLUSIONS}

In brief, a personalized medicine approach to pain management may be an effective tool to aid physicians in managing their patients' pain. Ideally, such an approach would also help optimize which medications patients are taking to minimize adverse interactions limiting effective treatment of chronic pain. Furthermore, understanding the personal genetic variation of those enzymes critically involved in narcotic metabolism may help identify those patients who are at higher risk for abusing opioids, in consideration with other biopsychosocial factors. Considering uncontrolled pain compromises immune function, promotes tumor growth, and is associated with increased morbidity and mortality, personalized and molecular medicine pain management may offer a novel way to help to improve the quality of life for those who suffer from inappropriately managed pain. ${ }^{23}$

\section{REFERENCES}

1. Schopflocher D, Taenzer P, Jovey R. The prevalence of chronic pain in Canada. Pain Res Manag. 2011;16:445-50.

2. Moulin DE, Clark AJ, Speechley M, et al. Chronic pain in Canada: prevalence, treatment, impact and the role of opioid analgesia. Pain Res Manag. 2002;7:179-84.

3. Boulanger A, Clark AJ, Squire P, et al. Chronic pain in Canada: have we improved our management of chronic noncancer pain? Pain Res Manag. 2007;12:39-47.

4. Todd KH, Ducharme J, Choiniere M, et al. Pain in the emergency department: results of the pain and emergency medicine initiative (PEMI) multicenter study. J Pain. 2007;8:460-6.

5. Bruehl S, Apkarian AV, Ballantyne JC, et al. Personalized medicine and opioid analgesic prescribing for chronic pain: opportunities and challenges. J Pain. 2013;14:103-13.

6. Ogino S, Galon J, Fuchs CS, et al. Cancer immunology-analysis of host and tumor factors for personalized medicine. Nat Rev Clin Oncol. 2011;8:711-9.

7. Van't Veer LJ, Bernards R. Enabling personalized cancer medicine through analysis of gene-expression patterns. Nature. 2008;452:564.

8. Yasui W, Oue Nm Aung PP, et al. Molecular-pathological prognostic factors of gastric cancer: a review. Gastric Cancer. 2005;8:86-94.

9. Stamer UM, Zhang L, Stüber F. Personalized therapy in pain management: where do we stand? Pharmacogenomics. 2010;11:843-64.

10. Kirchheiner, J. \& Brockmöller, J. Clinical consequences of cytochrome P450 2C9 polymorphisms. Clin Pharmacol Ther. 2005;77:1-16.

11. LaCroix-Fralish ML, Mogil JS. Progress in genetic studies of pain and analgesia. Annu Rev Pharmacol Toxicol. 2009;49:97-121.

12. Seeringer A, Kirchheiner J. Pharmacogenetics-guided dose modifications of antidepressants. Clin Lab Med. 2008;28:619-26.

13. Giummarra MJ, Gibson SJ, Allen AR, et al. Polypharmacy and chronic pain: harm exposure is not all about the opioids. Pain Med. 2015;16:472-9.

14. Hajjar ER, Cafiero, AC, Hanlon JT. Polypharmacy in elderly patients. Am J Geriat Psychiat. 2007;5:345-51.

15. Campa D, Gioia A, Tomei A, et al. Association of ABCB1/MDR1 and OPRM1 gene polymorphisms with morphine pain relief. Clin Pharmacol Ther. 2008;83:559-66.

16. Kim RB. Drugs as P-glycoprotein substrates, inhibitors, and inducers. Drug Metab Rev. 2002;34:47-54.

17. Wienkers LC, Heath TG. Predicting in vivo drug interactions from in vitro drug discovery data. Nat Rev Drug Discov. 2005;4:825.

18. Shi J, Hui L, Xu Y, et al. Sequence variations in the mu-opioid receptor gene (OPRM1) associated with human addiction to heroin. Human Mutat. 2002;19:459-60.

19. Kumar D, Chakraborty J, Das S. Epistatic effects between variants of kappa-opioid receptor gene and A118G of mu-opioid receptor gene increase susceptibility to addiction in Indian population. Prog Neuropsychopharmacol Biol Psychiatry. 2012;36:225-30.

20. Yuferov V, Levran O, Proudnikov D, et al. Search for genetic markers and functional variants involved in the development of opiate and cocaine addiction and treatment. Ann N Y Acad Sci. 2010;1187:184-207.

21. Joranson DE, Ryan KM, Gilson AM, et al. Trends in medical use and abuse of opioid analgesics. JAMA. 2000;283:1710-4.

22. Witkin LR, Diskina D, Fernandes S, et al. Usefulness of the opioid risk tool to predict aberrant drug-related behavior in patients receiving opioids for the treatment of chronic pain. J Opioid Manag. 2013;9:17787.

23. Lynch ME. The need for a Canadian pain strategy. Pain Res Manag. 2011;16:77-80.

24. National Report: Apparent opioid-related deaths (2016). Government of Canada. 